\title{
Intraocular Pressure (IOP) Changes after Posterior Nd-Yag Laser Capsulotomy
}

\section{Saley Hamidou Idrissa*, Bennis Ahmed, Chraibi Fouad, Abdellaoui Meriem and Benatiya Idriss Andaloussi}

Department of Ophthalmology, Sidi Mohamed Ben Abdellah University, FES,

Morocco

*Corresponding Author: Saley Hamidou Idrissa, Department of Ophthalmology, Sidi Mohamed Ben Abdellah University, FES, Morocco.
Received: September 29, 2021

Published: October 02, 2021

(C) All rights are reserved by Saley Hamidou Idrissa., et al.

\begin{abstract}
Introduction: The most common complication of cataract surgery is a secondary posterior capsule opacification (PCO). Its incidence is estimated at $37 \%$ in patients over 40 years of age. Nd-Yag laser capsulotomy is the treatment of choice for PCO but is known to be associated with complications such as increased intraocular pressure (IOP), cystoid macular edema, retinal detachment and corneal burns. The most common complication is increased IOP.

Aim: The aim of this study is to evaluate the changes in intraocular pressure (IOP) after Nd-Yag laser posterior capsulotomy (LPC) for PCO.

Patients and Methods: This is a longitudinal study in patients treated with posterior Nd-yag laser capsulotomy for secondary posterior capsule opacification in Hassan II hospital University of fez/Morocco. The inclusion criteria are: Patients older than 10 years, tonometry on the fellow (control) eye is possible, No history of glaucoma and endocular surgery, clear cornea, No pre-existing uveitis, IOP before Nd yag LPC is between $8 \mathrm{mmHg}$ and $21 \mathrm{mmHg}$. IOP was checked before laser, at one hour, 24 hours, one week, one month and three months after laser. The T student test is performed for statistical study; whose values are considered significant when the $\mathrm{p}$ is less than 0.05 .
\end{abstract}

Results: A total of 32 eyes from 30 patients were included in our study; 18 men and 14 women. The average age is $65.66 \pm 9$ years; $75 \%$ operated by extra-capsular extraction by phacoemulsification and $25 \%$ by manual extra-capsular extraction; All of our patients are pseudophakic. The average time between cataract surgery and Nd-yag LPC is 3 years. The mean IOP is: $13.66 \pm 1.7 \mathrm{mmHg}$ before posterior capsulotomy; then after the capsulotomy at 1 hour: $16.59 \pm 2.5 \mathrm{mmHg}$; at 1 day: $15.63 \pm 2.1 \mathrm{mmHg}$; at $7 \mathrm{days}: 14.28 \pm 1.7$ $\mathrm{mmHg}$; at 30 days: $13.84 \pm 1.5 \mathrm{mmHg}$; and at 90 days: $13.75 \pm 1.56 \mathrm{mmHg}$. The most used laser intensity is $2 \mathrm{mj}$ ( $31.3 \%$ of cases) with extremes of 1.8 to $3.2 \mathrm{mj}$. The most laser pulses interval used is 20 - 30 pulses (40.6\%) with extremes of 20 - 52 pulses.

The differences observed between the IOP before the Nd-yag laser capsulotomy and the IOP at 1 hour; at 1 day and 7 days after Nd-yag capsulotomy are statistically significant; whereas those observed between the IOP before the capsulotomy and the IOP on 30 days; and at 90 days are not statistically significant. We did not find a correlation between the increase in IOP and the intensity of the laser; also no correlation found between the number of pulses and the increase in IOP.

Conclusion: Nd-Yag LPC is a common procedure for treating secondary PCO after cataract surgery; and its most frequent complication remains the increase in IOP. In our series, the increase in IOP was significant within 7 days of capsulotomy and usually normalizes thereafter without any treatment. This increase in IOP was independent of the intensity of the pulse and the number of pulses.

Keywords: Intraocular Pressure (IOP); Posterior Capsule Opacification (PCO); Neodymium-Yttrium-Aluminum-Garnet Laser (Ndyag Laser), Nd-Yag Laser Posterior Capsulotomy (Nd-yag LPC); Extra-Capsular Cataract Extraction (ECCE) 


\section{Introduction}

The Nd-Yag L has been developed in Europe since the mid 1970s [1]. Today Nd-Yag LPC has become an established procedure for after cataract. Before the Nd-Yag laser came into use, the capsulotomy was done by performing a small puncture with a needle knife or 27 gauge needle, either at the time of original operation or as a secondary procedure through the limbus in aphakic or through pars plana in pseudophakic. The Nd-Yag $L$ preferred because it is non-invasive and infection cannot occur. The most important complication is a transient rise in IOP 1 - 3 hrs of Nd-Yag LPC. Occasionally the pressure rise is high and can cause serious damage to the optic nerve, so that the IOP should be monitored and appropriate measures should be taken if necessary [2]. In otherwise normal eyes, a mild elevation of IOP is of no consequence because it usually resolves within 24 hours especially when the patient receives anti-glaucoma drugs before and after laser application. However, in eyes with pre-existing glaucoma, the incidence of IOP elevation is higher and its duration is longer than in otherwise normal eyes. Some glaucomatous eyes may therefore require additional glaucoma therapy for several weeks following Nd-Yag LPC [3].

\section{Aim of the Study}

The aim of this study was to evaluate the changes of intraocular pressure (IOP) after Nd-Yag LPC for PCO at 1hour, 24 hour (1 day), one week (7 days), one month (30 days) and three months (90 days) in non glaucomatous patients.

\section{Patients and Methods}

This is a longitudinal study in patients treated with Nd-yag LPC for PCO. This is a study carried out over a period of 14 months; from October 2019 to December 2020 at the Hassan II Hospital university of Sidi Mohamed Ben Abdallah University in Fez/Morocco. Our inclusion criteria are:

- Patients older than 10 years;

- Patients not followed for glaucoma;

- No history of intraocular surgery in both eyes other than cataract surgery;

- Clear cornea;

- $\quad$ No pre-existing uveitis;

- Tonometry on the fellow (control) eye is possible;

- Only patients with posterior chamber IOL;

- $\quad$ IOP before Nd yag LPC is between $8 \mathrm{mmHg}$ and $21 \mathrm{mmHg}$.
VA is measured by the Snellen's chart before and 3 months after posterior Nd-yag LPC. The IOP is measured using the Goldmann applanation tonometer; with topical anesthesia oxybuprocaine $0.4 \%$ eye drops and fluorescein strips immediately before laser treatment and at 1 hour, 24 hours, one week, one month and 3 months after capsulotomy. The reported IOPs are the result of at least two measurements that have been averaged and corrected for pachymetry. All pupils were dilated with tropicamide $1 \%$ and/or neosynephrine $10 \%$ eye drops. The other elements collected are: the intensity of the laser and the number of laser pulses used during the capsulotomy.

The results were analyzed with

- Student-t-test: It is a statistical test used to signify the difference between the mean of 2 groups.

- Paired-t-test: It is used to signify the difference between paired values of the same group.

- P-Value: It is the probability which means if $\mathrm{P}<0.05$ or $\mathrm{P}<$ 0.001 the result of significant difference is not due to chance.

- P-value: $\mathrm{P}<0.05$ significant and $\mathrm{P}>0.05$ not significant.

\section{Results}

A total of 32 eyes from 30 patients were included in our study; 18 men and 14 women. The average age is $65.66 \pm 9$ years with extremes of 38 to 82 years. $75 \%$ operated by extra-capsular extraction by phacoemulsification and $25 \%$ by manual extra-capsular extraction; All of our patients are pseudophakic. The average time between cataract surgery and Nd-yag LPC is 3 years with extremes of 8 months to 7 years. Visual acuity before posterior capsulotomy is: $\leq 5 / 10$ in $87.5 \%$ and $<1 / 10$ in $40.6 \%$ of cases (Table 1 ). The mean IOP is: $13.66 \pm 1.7 \mathrm{mmHg}$ before posterior capsulotomy; then after the capsulotomy at one hour: $16.59 \pm 2.5 \mathrm{mmHg}$; at 1 day: $15.63 \pm 2.1 \mathrm{mmHg}$; at 7 days: $14.28 \pm 1.7 \mathrm{mmHg}$; at 30 days: 13.84 $\pm 1.5 \mathrm{mmHg}$; and at 90 days: $13.75 \pm 1.56 \mathrm{mmHg}$ (Table 2).

The differences observed between the IOP before the Nd-yag laser capsulotomy and the IOP at one hour; at 1 day and 7 days after capsulotomy are statistically significant ( $p<0.0001$ at $1 \mathrm{~h}, 1$ day and 7 days); but the differences observed between the IOP before the capsulotomy and the IOP at 30 days; and at 90 days are statistically not significant ( $\mathrm{p}=0.083$ at 30 days and $\mathrm{p}=0.414$ at 90 days).

We observe a strong rise in IOP one hour after Nd-yag LPC and which gradually decreases from 24 hours to 7 days to stabilize from 


\begin{tabular}{|l|c|c|}
\hline Visual Acuity & Numbers & Percentage \% \\
\hline $0,3 / 10$ & 2 & 6,3 \\
\hline $0,5 / 10$ & 5 & 15,6 \\
\hline $1 / 10$ & 6 & 18,8 \\
\hline $2 / 10$ & 5 & 15,6 \\
\hline $3 / 10$ & 4 & 12,5 \\
\hline $4 / 10$ & 3 & 9,4 \\
\hline $5 / 10$ & 3 & 9,4 \\
\hline $6 / 10$ & 4 & 12,5 \\
\hline Total & 32 & 100 \\
\hline
\end{tabular}

30 days to 90 days (Figure 1). The most widely used laser intensity is $2 \mathrm{mj}$ with extremes of 1.8 to $3.2 \mathrm{mj}$ (Figure 2). The relative effects of laser intensity on the rise in IOP at one hour after Nd-yag LPC are not significant $(\mathrm{p}=0,806)$. The most laser pulse interval used is 20 - 30 pulses (40.6\%) with extremes of 20-52 pulses (Figure 3); with the relative effects of Number of pulses on the rise in IOP at one hour after Nd-yag LPC which are not significant $(p=0,152)$. In our series, the technique of extracapsular cataract extraction (manual or phacoemulsification) does not influence the increase in IOP ( $p$ $=0.724$ ).

2 patients presented an increase in IOP on 1 day greater than 22

Table 1: VA before Nd-yag LPC. $\mathrm{mmHg}$ requiring a pressure-lowering medication by monotherapy

\begin{tabular}{|l|c|c|c|c|c|c|}
\hline & Mean (mmHg) & Median (mmHg) & SD (mmHg) & Minimum (mmHg) & Maximum (mmHg) & P-value \\
\hline IOP baseline & 13,66 & 13 & 1,696 & 11 & 18 & - \\
\hline IOP 1h & 16,59 & 16,5 & 2,538 & 12 & 23 & $<0,0001$ \\
\hline IOP 1 day & 15,63 & 16 & 2,121 & 12 & 12 & 18 \\
\hline IOP 7 days & 14,28 & 14,5 & 1,727 & 12 & 18 & $<0,0001$ \\
\hline IOP 30 days & 13,84 & 14 & 1,505 & 12 & 18 & 0,083 \\
\hline IOP 90 days & 13,75 & 13 & 1,566 & 12 & 0,414 \\
\hline
\end{tabular}

Table 2: Mean of IOP's.

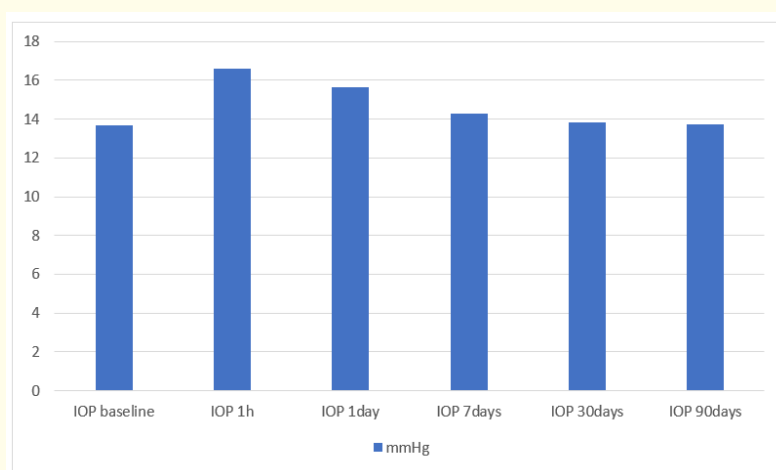

Figure 1: IOPs changes before and after laser.

with pressure balance at control on 7 days. Final corrected visual acuity is: $>6 / 10$ in $78.1 \%$ and $10 / 10$ in $25 \%$ of cases (Table 3).

\section{Discussion}

PCO is the most common late complication of uncomplicated ECCE. The older the patient at the time of surgery, the longer the

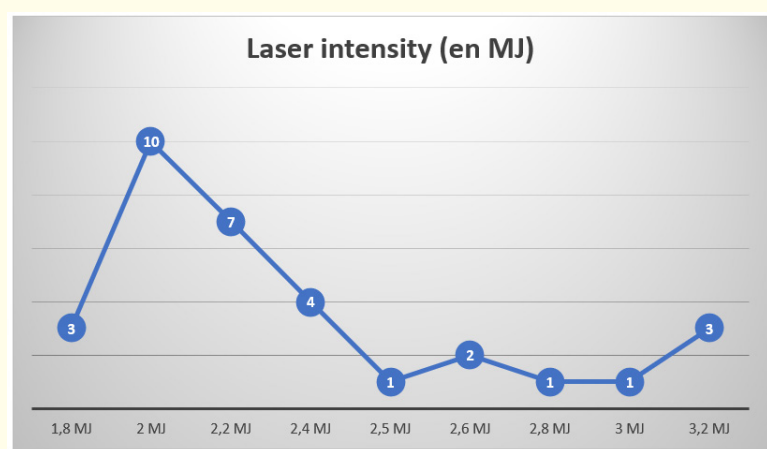

Figure 2: Laser intensities. period the patient was at risk for requiring posterior capsulotomy; This may be related to an age related decline in lenticular hyperplasia [3]. After-cataract has become a significant problem in that almost all pediatric patients and approximately half of adult patients develop an opaque posterior capsule after ECCE [8]. A number of methods may be used to avoid opacity of the posterior 


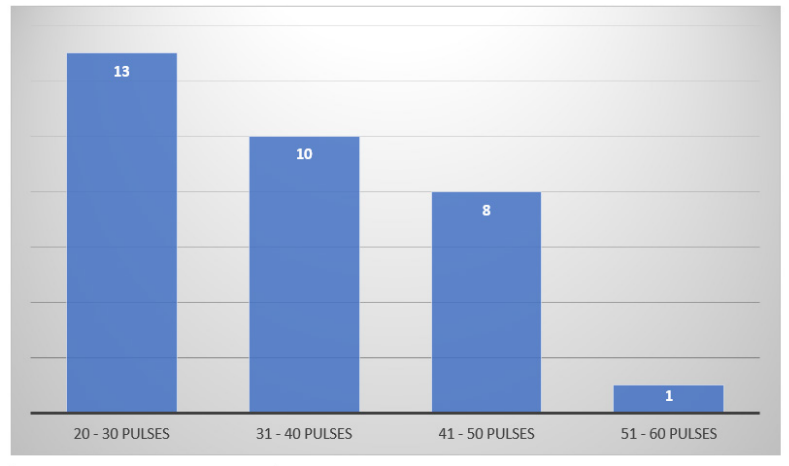

Figure 3: Number of laser pulses.

\begin{tabular}{|l|c|c|}
\hline Visual Acuity & Numbers & Percentage \% \\
\hline $2 / 10$ & 1 & 3,1 \\
\hline $4 / 10$ & 5 & 15,6 \\
\hline $6 / 10$ & 4 & 12,5 \\
\hline $7 / 10$ & 6 & 18,8 \\
\hline $8 / 10$ & 6 & 18,8 \\
\hline $9 / 10$ & 2 & 6,3 \\
\hline $10 / 10$ & 8 & 25,0 \\
\hline Total & 32 & 100 \\
\hline
\end{tabular}

Table 3: Final VA corrected.

capsule. They include polishing and vacuum cleaning of the posterior capsule. Various lens designs such as a laser ridge and convex surface posteriorly have been used to form mechanical barriers to the formation of Elschnig`s pearls [4]. The types of posterior capsule opacification are:

- Primary opacification: Some soft, clear cortex sticks to the posterior capsule (residual posterior subcapsular plaque). This becomes partially absorbed by the action of the aqueous but often becomes shut off by adhesion of the remains to posterior capsule [3].

- Elschnig's pearls: Are caused by proliferation of lens epithelium on to the posterior capsule at the site of apposition between remnants of anterior capsule and the posterior capsule [3] giving the posterior capsule a (fish eggs) appearance [5].

- Fibrous membrane: Consists of lens epithelium which has undergone fibrous metaplasia, these cells of epithelium con- gregate at the site of apposition between the anterior capsule remnant and the posterior capsule and can extend to a variable extent towards the visual axis. In posterior capsule with thicker fibrotic opacities, more power and shots may be needed to open the capsule [2].

Opacity seen with slit-lamp either by oblique lighting or retroillumination does not necessarily mean poor visual acuity [2]. The decision whether capsular opacity is significant requires the consideration of three factors:

- First: The slit-lamp appearance of opacification.

- Second: A fall of VA of 2 lines or more with the Snellen`s chart.

- Third: The clinical assessment of visual potential by evaluating the appearance of the macula [2].

The treatment of this secondary opacification of the posterior capsule is done with the Nd-yag laser. This is a solid state laser source emits an infra-red light invisible to the eye of $1064 \mathrm{~nm}$ wavelength. The Nd-yag laser in pulsed mode makes it possible to deliver a significant amount of energy in a very short time at a given point; It does not work by thermal effect, but by creating a very localized "plasma". It is useful for cutting or perforating at a very precise point, while respecting neighboring tissues. This is a non-invasive method of performing posterior capsulotomy on an outpatient basis [2].

The mechanism for rise in IOP is unknown, many explanations have been put forward to explain the transient rise in IOP following Nd-Yag LPC it may be caused by the following: *It has been suggested that aqueous debris (released lens material) may become trapped in the juxta-trabecular meshwork or in the internal pores of Schlemm's canal, causing increased outflow resistance $[6,7]$. *The shock-wave formed by the Nd-Yag Laser may induce a neurohumoral reflex, which causes the release of neuropeptides which account for the rise in IOP; ${ }^{*}$ Trabeculitis secondary to the radiating shock-wave [10]. *Occasionally, the IOP rise can be due to pupillary block after the Nd-Yag LPC has displaced the anterior vitreous face [2].

Our series shows a significant difference in the increase in IOP after posterior Nd-yag LPC at 1 hour, 1 day and 7 days; however, this increase is not significant at 30 days and 90 days. Our results are consistent with those obtained in 2016 by Niharika and $\mathrm{Al}$ [8] with a significant increase in IOP at 1 hour and 7 days. Also our results are consistent with those obtained in 2016 by Yilmaz [9] at 
30 days and 90 days. In our study, 2 patients had presented an IOP greater than $22 \mathrm{mmHg}$ one hour after the laser, explained by an IOP before the capsulotomy which was greater than $18 \mathrm{mmHg}$, requiring a hypothonizing monotherapy based on beta-blocker with IOP normalized to the control to 7 days.

Laser intensity was not a factor favoring the increase in IOP in our series, which is consistent with the series by Niharika and al [8]. The number of laser pulses is also not a factor influencing the increase in IOP in our series as is the case of the results of Yilmaz [9] for less than 40 pulses.

In our series, the ECCE technique (manual or phacoemulsification) is not a protective factor in elevation of IOP; This is not in accordance with a study carried out in 1997 by Ronald R. Holweger [11] which reports that in eyes operated by the phacoemulsification technique, lack of communication between the aqueous and vitreous compartments with an intact capsulorhexis over the lens optic rim prevented obstruction of the trabecular meshwork by cellular debris or vitreous, thus avoiding a significant IOP elevation. In some studies, including that performed in 1986 by Boen-Tan [7], pretreatment with $0.5 \%$ timolol eye drops has been used to minimize the increase in IOP but it does not provide complete protection. In our series; glaucomatous patients were not included; they are more likely to require long-term complementary treatment than non-glaucomatous patients after laser capsulotomy [12].

\section{Conclusion}

Nd-Yag LPC is a common procedure for treating secondary PCO after cataract surgery; and its most frequent complication remains the increase in IOP. In our series, the increase in IOP was significant within 7 days of capsulotomy and usually normalizes thereafter without any treatment. This increase in IOP was independent of the intensity of the pulse and the number of pulses. This study shows the importance of measuring IOP before and after Nd-yag LPC in all patients. A 2 - $3 \mathrm{~mm}$ capsulotomy is usually sufficient to improve the VA and minimize the increase in IOP.

\section{Bibliography}

1. MJ Roper Hall. "Stallard's eye surgery". Seventh edition (1989): 30-31.

2. Ian J Constable. "Arther Siew Ming Lim. Laser its clinical uses in eye diseases". Second edition (1990): 166-170.

3. Jack J Kanski. "Clinical ophthalmology. A systemic approach". Third edition (1995): 304-305.
4. Daniel Vaughan., et al. "General ophthalmology". Twelfth edition (1989): 416-417.

5. Stephen JH Miller. "Parson's diseases of the eye seventeenth edition" (1984): 179.

6. Kraff MC., et al. "Intraocular Pressure and the corneal endothelium after Nd-Yag laser posterior capsulotomy. Relative effect of aphakia and pseudophakia". Archives of Ophthalmology 103.4 (1985): 511-514.

7. Boen Tan TN and Stilma-JS. "Prevention of IOP-rise following $\mathrm{Nd}$-Yag laser posterior capsulotomy with preoperative timolol eye drops and 1 tablet acetazolamide 250mg systematically". Documenta Ophthalmologica 64.1 (1986): 59-67.

8. Niharika K Sketty and Sridhar. "Study of variation in intraocular pressure spike (IOP) following Nd-yag laser capsulotomy". Journal of Clinical and Diagnostic Research 10.12 (2016): 9-12.

9. Yilmaz Tolga. "Long-term changes in subfoveal choroidal thickness and central thickness after Nd-yag laser capsulotomy". International Ophthalmology 37.4 (2016): 1003-1008.

10. Van-der Feltz-Van-der-Sloot-D., et al. "Prevention of IOP-rise following Nd-Yag laser capsulotomy with topical timolol and indomethacin". Documenta Ophthalmologica 70.2-3 (1988): 209-214.

11. Ronald R Holweger. "Intraocular pressure changes after NdYag capsulotomy". The Journal of Cataract and Refractive Surgery 23 (1997): 115-121.

12. Jayne Ge. "Long-term effect of Nd-Yag LPC on IOP”. Archives of Ophthalmology 118 (2000): 1334-1337.

\section{Volume 4 Issue 11 November 2021}

(C) All rights are reserved by Saley Hamidou Idrissa., et al. 